\title{
Overall Profile of a Pediatric Multidisciplinary Feeding Clinic
}

\author{
Ji Sun Jung, $\mathrm{MD}^{1}$, Hyun Jung Chang, $\mathrm{MD}^{2}$, Jeong-Yi Kwon, $\mathrm{MD}^{1}$
}

\begin{abstract}
${ }^{1}$ Department of Physical and Rehabilitation Medicine, Samsung Medical Center, Sungkyunkwan University School of Medicine, Seoul; ${ }^{2}$ Department of Physical Medicine and Rehabilitation, Samsung Changwon Hospital, Sungkyunkwan University School of Medicine, Changwon, Korea
\end{abstract}

\begin{abstract}
Objective To evaluate the overall profile of children with feeding disorders and their relationships to medical conditions in an outpatient feeding clinic of a tertiary hospital.

Methods The medical records of 143 children who had visited the feeding clinic between January 2010 and June 2014 were reviewed retrospectively. The presence of a feeding disorder (feeding behavior disorder, dysphagia, and/or failure to thrive [FTT]) and the children's medical conditions were examined by a physiatrist.

Results Half of the patients $(\mathrm{n}=74,51.7 \%)$ were under 15 months of age, and 68 (47.6\%) were born preterm. Ninety-three patients $(65.0 \%)$ met the criteria for any combination of feeding behavior disorder, dysphagia, or FTT. Cardiorespiratory disease was the most common medical condition; children with this condition were more likely to show sensory food aversion and FTT. Feeding behavior disorders were significantly associated with gastrointestinal problems, and dysphagia was significantly related to almost all medical conditions.

Conclusion A multidisciplinary approach to children with feeding difficulties is proposed for comprehensive evaluation and treatment because combinations of feeding problems are very common among children. This overall profile could provide clinicians with a clear understanding of the complexity of feeding disorders and their relationships with various medical conditions in children.
\end{abstract}

Keywords Feeding behavior, Failure to thrive, Dysphagia, Comorbidity

\section{INTRODUCTION}

Feeding problems occur in $25 \%-45 \%$ of typically developing children and $80 \%$ of those with developmental delays due to chronic medical conditions $[1,2]$. Increased survival of sick and preterm infants may be associated with long-term medical and developmental problems, such as feeding difficulties in children and emotional problems in families [3,4].

Children with feeding difficulties usually have coexist-

Received August 24, 2015; Accepted January 26, 2016

Corresponding author: Jeong-Yi Kwon

Department of Physical Medicine and Rehabilitation, Samsung Medical Center, Sungkyunkwan University School of Medicine, 81 Irwon-ro, Gangnamgu, Seoul 06351, Korea. Tel: +82-2-3410-2818, Fax: +82-2-3410-0052, E-mail: jeongyi.kwon@samsung.com

ORCID: Ji Sun Jung (http://orcid.org/0000-0001-8579-5794); Hyun Jung Chang (http://orcid.org/0000-0001-9126-4316); Jeong-Yi Kwon (http://orcid. org/0000-0003-2011-8834).

(c) This is an open-access article distributed under the terms of the Creative Commons Attribution Non-Commercial License (http://creativecommons.org/ licenses/by-nc/4.0) which permits unrestricted noncommercial use, distribution, and reproduction in any medium, provided the original work is properly cited. Copyright (c) 2016 by Korean Academy of Rehabilitation Medicine 
ing medical, behavioral, psychological, and developmental problems; these conditions rarely function singly or independently. Berlin et al. [5] reported that $48 \%-85 \%$ of children with feeding difficulties have 2 more conditions (developmental, behavioral, and/or medical). Moreover, Benoit et al. [6] assessed the efficacy of behavioral interventions in eliminating the need for enteral tube feeding in infants compared with only nutritional treatment and found success among $47 \%$ of the 32 subjects in the behavioral group. Burklow et al. [7] presented a classification system for complex pediatric feeding disorders and proposed that feeding problems occur because of interactions between biological and behavioral factors. Rommel et al. [8] reported that children with gastrointestinal problems have a variety of oropharyngeal dysfunctions and children with heart disease have abnormal tactile responses. In addition, isolated neurological disorders are correlated with oral motor problems. However, few studies have shown the relationships between medical conditions and feeding disorders in medically complicated children.

A better understanding of the relationships between feeding disorders and their underlying medical diseases will aid in timing effective interventions for children with feeding difficulties. Therefore, the purpose of this study was to evaluate the overall profile of children with feeding disorders and their relationships to medical conditions in an outpatient feeding clinic of a tertiary hospital.

\section{MATERIALS AND METHODS}

\section{Subjects}

The enrolled children $(n=152)$ were all under 6 years of age and had visited the pediatric feeding clinic in a tertiary university hospital from January 2010 to June 2014, and all were reviewed retrospectively; patients were referred with complaints of food refusal, selective eating, aspiration, gagging, and growth faltering. Nine patients were excluded because insufficient clinical information was available. This study was approved by the Institutional Review Board of Samsung Medical Center.

\section{Methods}

Demographic findings, growth profile at the first clinic visit, developmental history, and feeding history were reviewed for all patients. The presence of feeding disorder (feeding behavior disorders, dysphagia, and/or failure to thrive [FTT]) and the children's medical conditions were examined by a physiatrist. FTT is a state of inadequate growth or the inability to maintain growth, usually in early childhood [9]; it was defined as weight-for-age and/ or height-for-age and/or head circumference-for-age under the 5th percentile [10]. Dysphagia was defined as problems with swallowing, documented by history of aspiration pneumonia, risk for aspiration (e.g., coughing, increased work of breathing) and/or identified oral- and pharyngeal-phase abnormalities via videofluoroscopic swallowing study [5].

\section{Feeding behavioral disorders}

The diagnoses of six feeding behavior disorder subtypes were made by a clinician based on the Diagnostic and Statistical Manual of Mental Disorders, Fifth Edition criteria for Feeding Disorders of Infancy and Early Childhood [11]. Children can have a combination of the six disorder subtypes (Appendix 1).

\section{Medical diagnostic categories}

Based on a previous study by Burklow et al. [7], we classified the medical diagnostic categories as neurological, gastrointestinal, cardiorespiratory, ear-nose-throat (ENT), and metabolic disease. A patient could have more than one medical diagnosis; therefore, the medical diagnostic categories were not reciprocally exclusive.

\section{Statistical analysis}

The statistical analysis was conducted with SPSS ver. 20.0 statistical software (IBM, Armonk, NY, USA). Frequency analysis and descriptive statistics were used to evaluate demographic characteristics. The chi-square test for a $2 \times 2$ table was used to identify the relationships between medical conditions and feeding disorders. A pvalue less than 0.05 was considered significant.

\section{RESULTS}

\section{Demographic characteristics and reasons for the referrals}

Mean age at the first visit was 17.6 \pm 11.2 months. Half of the subjects $(n=74,51.7 \%)$ were less than 15 months of age. Weight-for-age, height-for-age, and/or head circumference-for-age under the 5 th percentile among children identified as FTT ( $\mathrm{n}=93,65.0 \%)$ were observed in 41 
(44.1\%), 17 (18.3\%), and 35 (37.6\%) patients, respectively. The reasons for visiting the feeding clinic were growth faltering ( $\mathrm{n}=67,46.9 \%)$, swallowing difficulties $(\mathrm{n}=59$, $41.3 \%)$, picky eating $(n=53,37.1 \%)$, and oral aversion $(\mathrm{n}=49,34.3 \%)$. Growth faltering $(\mathrm{n}=54,50.5 \%)$ was the most common reason for visiting the clinic in children younger than 2 years of age, whereas picky eating $(n=19$, $52.8 \%$ ) was the most common reason for those over age 2. Sixty-eight patients born prematurely were reported to have FTT $(51,75.0 \%)$, dysphagia (31, 45.6\%), and feeding behavior disorder $(47,69.1 \%)$. Thirteen $(9.1 \%)$ were fed by non-oral feeding methods such as a nasogastric tube, gastrostomy, and jejunostomy (Table 1). Of the 68 subjects with a surgical history, 37 (54.4\%) had heart, gastrointestinal, or brain surgery, and 15 had undergone 2 or more surgeries. Others included laryngomicroscopic surgery, tonsillectomy, repair of inguinal hernia, correc- tion of cleft lip, and diaphragm plication (Table 2).

\section{Medical conditions associated with feeding disorders}

Cardiorespiratory disease (42 of 143, 29.4\%) was the most frequently seen in children with multiple medical conditions. Among 52 (36.4\%) children with only 1 condition, cardiorespiratory disease was noted in 18 (34.6\%), followed by gastrointestinal (13 of 52, 25.0\%) and neurological (13 of 52, 25.0\%) diseases. Twenty-one children had 2 medical diseases, with the highest being cardiorespiratory-neurologic $(7,33.3 \%)$, cardiorespiratory-gastrointestinal $(4,19.0 \%)$, and cardiorespiratory-metabolic $(3,14.3 \%)$ diseases. Eight children had 3 diseases, and 3 children had 4 diseases. We found no underlying medical disorder to explain the feeding disorders in 59 (41.3\%) of the 143 subjects (Table 3 ).

Table 1. Demographic characteristics of the children ( $n=143)$

\begin{tabular}{|cc|}
\hline \multicolumn{1}{c}{ Characteristic } & Value \\
\hline Gender & $74(51.7)$ \\
\hline Boy & $69(48.3)$ \\
\hline Girl & $17.6 \pm 11.2(0-69)$ \\
\hline Corrected age at presentation (mo) & $52(36.4)$ \\
\hline$<1$ year & $55(38.5)$ \\
\hline $1-2$ years & $36(25.2)$ \\
\hline $2-7$ years & $34.5 \pm 6.3(23.1-41.1)$ \\
\hline Gestational age (wk) & $68(47.6)$ \\
\hline Preterm (<37 weeks) & $75(52.4)$ \\
\hline Full-term ( $\geq 37$ weeks) & $62(43.4)$ \\
\hline Birth weight (g) & $81(56.6)$ \\
\hline$<2,500$ & $93(65.0)$ \\
\hline$\geq 2,500$ & 41 \\
\hline FTT & 17 \\
\hline Total & $354.6 \pm 1,008.1(470-4,700)$ \\
\hline Weight-for-age under 5th percentile & \\
\hline Weight-for-age and height-for-age under 5th percentile & $11(7.7)$ \\
\hline Weight-for-age, height-for-age, and head circumference-for-age & $19(83.2)$ \\
\hline under 5th percentile & \\
\hline Diet status & \\
\hline Tube feeding & \\
\hline Oral feeding & \\
\hline Tube+Oral & \\
\hline
\end{tabular}

Values are presented as numbers (\%) or mean \pm standard deviation (range).

FTT, failure to thrive. 
Subtypes of feeding behavior disorders by medical condition

Neurological disorders were significantly associated with posttraumatic feeding disorder $(\mathrm{p}<0.05)$, and gastrointestinal disorders were significantly related to feeding disorders associated with a concurrent medical condition $(\mathrm{p}<0.05)$. Cardiorespiratory disorders were correlated with sensory food aversion $(\mathrm{p}<0.05)$. Among children with no medical condition, 49 had feeding behavior disorders with and without FTT $(57.1 \%$ and $42.9 \%$, re-

Table 2. Subjects with surgical histories $(\mathrm{n}=68)$

\begin{tabular}{|cc}
\hline & No. of patients (\%) \\
\hline Cardiac & $20(29.4)$ \\
\hline $\begin{array}{l}\text { Surgical ligation of } \\
\text { patent ductus arteriosus }\end{array}$ & 7 \\
\hline Congenital heart surgery & 13 \\
\hline Gastrointestinal & $12(17.6)$ \\
\hline Bowel resection or enterostomy & 7 \\
\hline Fundoplication & 5 \\
\hline Brain & $5(7.4)$ \\
\hline Shunt & 4 \\
\hline Navigation biopsy & 1 \\
\hline Others & $16(23.5)$ \\
\hline Two or more surgeries & $15(22.1)$ \\
\hline
\end{tabular}

Others included laryngomicroscopic surgery, tonsillectomy, repair of inguinal hernia, correction of cleft lip, and diaphragm plication. spectively). Many of these children had feeding behavior disorders such as sensory food aversion $(31,63.3 \%)$ and infantile anorexia $(15,30.6 \%)$ (Table 4$)$.

Correlations between FTT, dysphagia, and feeding behavior disorders with a medical condition

FTT was more common in children with cardiorespiratory disease $(\mathrm{p}<0.05)$, and dysphagia was frequently accompanied by neurological, cardiorespiratory, and ENT diseases. Feeding behavior disorders were more frequent in children with gastrointestinal disease $(p<0.05)$. Nine of the 59 children with no medical condition had dysphagia (Table 5).

Table 3. Associated medical conditions ( $\mathrm{n}=143)$

\begin{tabular}{|lc|}
\hline & No. of patients \\
\hline One medical condition & 18 \\
\hline Cardiorespiratory disease & 13 \\
\hline Neurological disease & 13 \\
\hline Gastrointestinal disease & 6 \\
\hline ENT disease & 2 \\
\hline Metabolic disease & 21 \\
\hline Two medical conditions & 8 \\
\hline Three medical conditions & 3 \\
\hline Four medical conditions & 59 \\
\hline No medical conditions & \\
\hline
\end{tabular}

ENT, ear-nose-throat.

Table 4. Subtypes of feeding behavior disorders by medical condition

\begin{tabular}{|c|c|c|c|c|c|c|}
\hline \multirow[b]{2}{*}{ Medical conditions } & \multicolumn{6}{|c|}{ Feeding behavior disorders $(n=104)$} \\
\hline & $\begin{array}{c}\text { FD of } \\
\text { state } \\
\text { regulation } \\
(n=5)\end{array}$ & $\begin{array}{c}\text { FD of } \\
\text { caregiver- } \\
\text { infant } \\
\text { reciprocity } \\
(n=2)\end{array}$ & $\begin{array}{c}\text { Infantile } \\
\text { anorexia } \\
(n=28)\end{array}$ & $\begin{array}{c}\text { Sensory } \\
\text { food } \\
\text { aversion } \\
(n=60)\end{array}$ & $\begin{array}{c}\text { Posttraumatic } \\
\text { FD, } \\
\text { feeding } \\
\text { phobia } \\
(n=15)\end{array}$ & $\begin{array}{l}\text { FD associated } \\
\text { with a } \\
\text { concurrent } \\
\text { medical } \\
\text { condition } \\
(n=23)\end{array}$ \\
\hline Neurological disease & 2 & 0 & 3 & 12 & $7^{*}$ & 11 \\
\hline Gastrointestinal disease & 1 & 0 & 7 & 14 & 5 & $15^{* *}$ \\
\hline Cardiorespiratory disease & 2 & 0 & 10 & $12^{*}$ & 6 & 11 \\
\hline ENT disease & 0 & 0 & 0 & 4 & 3 & 4 \\
\hline Metabolic disease & 1 & 0 & 2 & 1 & 0 & 3 \\
\hline None & 2 & 2 & 15 & 31 & 3 & 0 \\
\hline
\end{tabular}

Values are presented as the numbers of children.

Numbers do not add up to $100 \%$ because children can have more than one type of feeding behavior disorders.

FD, feeding disorder; ENT, ear-nose-throat.

${ }^{*} \mathrm{p}<0.05,{ }^{* *} \mathrm{p}<0.01$ by chi-square test with $2 \times 2$ tables. 
Table 5. Correlations between FTT, dysphagia, and feeding behavior disorders in children with a medical condition or not

\begin{tabular}{lccc}
\hline Medical conditions & FTT & Dysphagia & Feeding behavior disorders \\
\hline Neurological disease & 25 & $27^{* *}$ & 22 \\
\hline Gastrointestinal disease & 19 & 15 & $28^{* *}$ \\
\hline Cardiorespiratory disease & $34^{*}$ & $25^{*}$ & 27 \\
\hline ENT disease & 6 & $10^{*}$ & 7 \\
Metabolic disease & 8 & 7 & 6 \\
\hline None & 35 & $9^{* *}$ & $49^{*}$ \\
\hline
\end{tabular}

FTT, failure to thrive; ENT, ear-nose-throat.

${ }^{*} \mathrm{p}<0.05,{ }^{* *} \mathrm{p}<0.01$ by chi-square test with $2 \times 2$ tables.

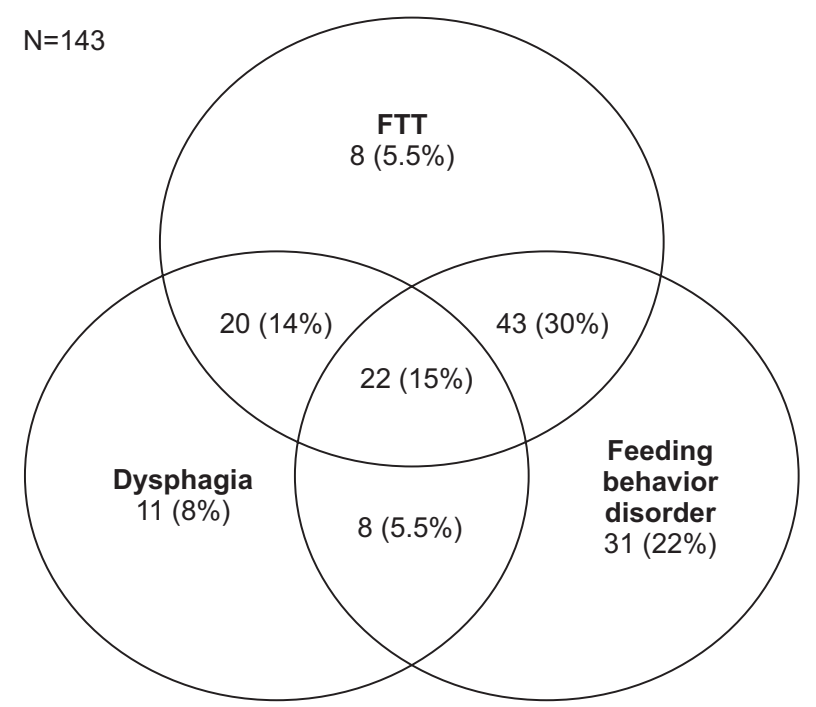

Fig. 1. Proportions of patients who present with failure to thrive (FTT), dysphagia, and a feeding behavior disorder.

A total of $104(72.7 \%)$ children had a feeding behavior disorder, 93 (65.0\%) had FTT, and 61 (42.7\%) had dysphagia. These problems occurred alone (feeding behavior disorders in $22 \%$, FTT in $5.5 \%$, and dysphagia in $8 \%$ ) and in combination. The combination of FTT-feeding behavior disorder was identified most frequently $(n=43,30 \%)$, followed by FTT-dysphagia $(n=20,14 \%)$ and FTT-dysphagia-feeding behavior disorder $(n=22,15 \%)$ (Fig. 1).

\section{DISCUSSION}

The present study provides the demographic characteristics of children with feeding difficulties and describes the complex nature of feeding disorders in these children. These results will be very informative for clinicians in providing proper diagnoses and interventions to chil- dren with feeding disorders.

Approximately half of our subjects were younger than 15 months of age, which corresponded to the results of a study by Rommel et al. [8]. Growth faltering was the primary cause of the visit to the feeding clinic in children younger than 2 years of age, whereas picky eating was the major cause in children older than age 2. Food refusal and food selectivity typically appear during feeding method transitions such as from bottle to spoon feeding and acquisition of the self-feeding skill [11], and the prevalence of picky eating increases to $50 \%$ at $19-24$ months of age [12]. Children between 6 months and 3 years of age are negotiating between autonomy and dependence on their parents [13]. The growth of a child's oral motor structure occurs at around 24 months to provide proper chewing skills [14], and children are gradually introduced to solid and viscous textured foods. If a child has difficulty processing food, it can result in oral hypersensitivity and oral-motor dysfunction $[15,16]$.

In the present study, $47.6 \%$ (68/143) of children had undergone 1 or more surgeries. Food refusal may follow a trauma to the oropharynx or gastrointestinal tract [17]. Children with a history of surgery for cleft palate can develop dysphagia and learned avoidance patterns [18], and dysphagia can appear following open heart surgery or prolonged endotracheal intubation $[19,20]$. There is intestinal rehabilitation available for patients with intestinal failure due to bowel resection that attempts to enhance the intestinal adaptive process and restore enteral anatomy [21]. It is important to have access to surgical histories because surgery can alter feeding routes and change feeding milestones.

In this study, the most common medical disorder that accompanied a feeding disorder in children was cardio- 
respiratory disease, followed by neurological and gastrointestinal diseases. These results correspond to those of a previous study on infants younger than 1 year of age with dysphagia [22], although Burklow et al. [3] reported that neurological disease was the most common accompanying disease in children under 10 years of age with feeding difficulties. Furthermore, Rommel et al. [8] reported that gastrointestinal disease was the most frequent medical diagnosis in children under age 10 who were examined for severe feeding problems. These differences between studies may result from the age ranges of the subjects enrolled, the compositions of the underlying medical diseases, or referral resources. We enrolled children under 6 years of age, and their mean age was less than that of previous studies.

Cardiorespiratory problems such as congenital heart disease and respiratory distress syndrome were significantly related to sensory food aversion in this study, and this finding is consistent with those of a previous study [8]. Complicated and coordinated sucking, swallowing, and breathing skills are required for children to feed orally [23], and it is possible that children with cardiorespiratory compromise tend to have difficulties with wellcoordinated suck-swallowing-breathing patterns, which may lead to aspiration, limited oral intake, and impaired sensory processing of food [24]. Medical conditions associated with inadequate caloric absorption (e.g., chronic diarrhea and metabolic disorders) or excessive caloric expenditure (e.g., hyperthyroidism and cleft lip and palate) may result in FTT [9]; however, only cardiorespiratory conditions were statistically related to FTT in this study. This finding may be attributed to the high ratio of children with cardiopulmonary disease and the result of comprehensive nutritional support team work in our hospital. Growth failure in children with cardiorespiratory disease is related to poor feeding, increased metabolic rate, malabsorption, and breathlessness [25]. Therefore, early comprehensive feeding evaluation and intervention are recommended for children with cardiorespiratory disease, particularly in those who have undergone major surgery. Nutrition counseling is critical, and programs for parents can also be developed to reduce children's sensory defensiveness with regard to the textures, tastes, temperatures, and smells of new foods.

Various feeding behavior disorders have been reported in children with medical diseases, and Kerzner et al. [26] suggested that it is necessary to pay attention to behavioral symptoms and signs to recognize feeding difficulties; It is important to identify behavioral red flags such as food fixation (selective, extreme dietary limitations), noxious (forceful and/or persecutory) feeding, abrupt cessation of feeding after a triggering event, anticipatory gagging, and FTT. In this study, $65.5 \%$ (55/84) of children with a medical condition also had 1 or more feeding behavior disorders, and one study reported that the presence of a medical condition did not exclude the effects of considerable behavioral components in $85 \%$ of children who presented with feeding difficulties [7]. Complex feeding problems require that clinicians carefully recognize a variety of components, including developmental and feeding history, medical conditions, emotional status, and nutrition. Through this approach, it is possible to identify the origin of a feeding problem and promote the most effective treatment plan [27].

Notably, we found no underlying medical disorder to explain the feeding disorders in 59 (41.3\%) of the $143 \mathrm{sub-}$ jects; many of these children had feeding behavior disorders such as sensory food aversion and infantile anorexia with FTT. This group of patients is part of the majority in our feeding clinic (FTT-feeding behavior disorders, $41.1 \%)$. Although transient picky eating may be normal in children and disappear following development [28], special attention should be given to prevent overlooking detrimental effects to a child's early development. Children with nonorganic FTT and a feeding problem have more sensory processing difficulties and delays in cognition, motor skills, and language development compared with those of age-matched controls [29].

In addition, we observed that 9 children with no medical condition had dysphagia, which we defined as a chewing and swallowing problem based on reports that included coughing, increased work of breathing, history of aspiration pneumonia, and/or abnormal results of a videofluoroscopic swallowing study. These children had difficulties chewing and moving solid food to the oropharynx or esophagus during the transition from liquid to solid feeding, and this result suggests that the causes of dysphagia may include not only medical diseases such as neurological or structural abnormalities but also psychogenic or unknown factors [30].

Ninety-three patients $(65.0 \%)$ met the criteria for any combination of 2 or more of a feeding behavior disorder, 
dysphagia, or FTT. Managing children with feeding difficulties may vary depending on the clinical setting and the training backgrounds of the medical personnel. For instance, pediatric gastroenterologists focus on diagnosing possible organic dysfunction and assessing nutritional status $[26,31]$, pediatric psychologists play a major role in treating patients with feeding behavior disorders and in the interactions between caretakers and children, and physiatrists are more attentive to diagnosing and managing oropharyngeal dysphagia. Therefore, the complex nature of feeding disorders in children requires a multidisciplinary team approach.

A number of limitations of this study should be mentioned. Because the data were collected retrospectively from a single feeding clinic in a tertiary hospital, our findings do not necessarily represent the wide spectrum of children with feeding difficulties. Moreover, many other important aspects of feeding disorders including child-parent interactions and development were not explored. In addition, although our study suggested that it is important to have access to surgical histories, our data were insufficient for analyzing any relationships between surgery history and feeding disorders because the types of surgery varied and our sample was somewhat small. A prospective study that includes more detailed evaluations of nutrition, sensory defensiveness, oral motor function, development assessment, and age-appropriate feeding skills is needed in children with feeding disorders.

In conclusion, a multidisciplinary team approach is proposed to comprehensively evaluate and treat children with feeding difficulties because combinations of feeding problems are very common among children. This overall profile will provide clinicians with a clear understanding of the complexity of feeding disorders in children and their relationships with various medical conditions.

\section{CONFLICT OF INTEREST}

No potential conflict of interest relevant to this article was reported.

\section{REFERENCES}

1. Manikam R, Perman JA. Pediatric feeding disorders. J Clin Gastroenterol 2000;30:34-46.

2. Lefton-Greif MA, Arvedson JC. Pediatric feeding and swallowing disorders: state of health, population trends, and application of the international classification of functioning, disability, and health. Semin Speech Lang 2007;28:161-5.

3. Burklow KA, McGrath AM, Valerius KS, Rudolph C. Relationship between feeding difficulties, medical complexity, and gestational age. Nutr Clin Pract 2002;17:373-8.

4. Jarjour IT. Neurodevelopmental outcome after extreme prematurity: a review of the literature. Pediatr Neurol 2015;52:143-52.

5. Berlin KS, Lobato DJ, Pinkos B, Cerezo CS, LeLeiko NS. Patterns of medical and developmental comorbidities among children presenting with feeding problems: a latent class analysis. J Dev Behav Pediatr 2011;32:41-7.

6. Benoit D, Wang EE, Zlotkin SH. Discontinuation of enterostomy tube feeding by behavioral treatment in early childhood: a randomized controlled trial. J Pediatr 2000;137:498-503.

7. Burklow KA, Phelps AN, Schultz JR, McConnell K, Rudolph C. Classifying complex pediatric feeding disorders. J Pediatr Gastroenterol Nutr 1998;27:143-7.

8. Rommel N, De Meyer AM, Feenstra L, VeeremanWauters $\mathrm{G}$. The complexity of feeding problems in 700 infants and young children presenting to a tertiary care institution. J Pediatr Gastroenterol Nutr 2003;37:75-84.

9. Cole SZ, Lanham JS. Failure to thrive: an update. Am Fam Physician 2011;83:829-34.

10. Behrman RE, Kliegman R, Nelson WE. Nelson textbook of pediatrics. Philadelphia: W.B. Saunders; 1996.

11. Chatoor I. Diagnosis and treatment of feeding disorders in infants, toddlers, and young children. Washington: Zero to Three; 2009.

12. Carruth BR, Ziegler PJ, Gordon A, Barr SI. Prevalence of picky eaters among infants and toddlers and their caregivers' decisions about offering a new food. J Am Diet Assoc 2004;104(1 Suppl 1):s57-64.

13. Steinberg C. Feeding disorders of infants, toddlers, and preschoolers. B C Med J 2007;49:183-6.

14. Gisel EG. Effect of food texture on the development of chewing of children between six months and two years of age. Dev Med Child Neurol 1991;33:69-79.

15. Coulthard H, Harris G, Emmett P. Delayed introduction of lumpy foods to children during the comple- 
mentary feeding period affects child's food acceptance and feeding at 7 years of age. Matern Child Nutr 2009;5:75-85.

16. Bernard-Bonnin AC. Feeding problems of infants and toddlers. Can Fam Physician 2006;52:1247-51.

17. Chatoor I, Ganiban J, Harrison J, Hirsch R. Observation of feeding in the diagnosis of posttraumatic feeding disorder of infancy. J Am Acad Child Adolesc Psychiatry 2001;40:595-602.

18. Field D, Garland M, Williams K. Correlates of specific childhood feeding problems. J Paediatr Child Health 2003;39:299-304.

19. Kogon BE, Ramaswamy V, Todd K, Plattner C, Kirshbom PM, Kanter KR, et al. Feeding difficulty in newborns following congenital heart surgery. Congenit Heart Dis 2007;2:332-7.

20. Barker J, Martino R, Reichardt B, Hickey EJ, RalphEdwards A. Incidence and impact of dysphagia in patients receiving prolonged endotracheal intubation after cardiac surgery. Can J Surg 2009;52:119-24.

21. DiBaise JK, Young RJ, Vanderhoof JA. Intestinal rehabilitation and the short bowel syndrome: part 2. Am J Gastroenterol 2004;99:1823-32.

22. Uhm KE, Yi SH, Chang HJ, Cheon HJ, Kwon JY. Videofluoroscopic swallowing study findings in full-term and preterm infants with dysphagia. Ann Rehabil Med 2013;37:175-82.

23. Verhagen JV, Engelen L. The neurocognitive bases of human multimodal food perception: sensory integration. Neurosci Biobehav Rev 2006;30:613-50.

24. Davis AM, Bruce AS, Khasawneh R, Schulz T, Fox C,
Dunn W. Sensory processing issues in young children presenting to an outpatient feeding clinic. J Pediatr Gastroenterol Nutr 2013;56:156-60.

25. Forchielli ML, McColl R, Walker WA, Lo C. Children with congenital heart disease: a nutrition challenge. Nutr Rev 1994;52:348-53.

26. Kerzner B, Milano K, MacLean WC Jr, Berall G, Stuart S, Chatoor I. A practical approach to classifying and managing feeding difficulties. Pediatrics 2015;135:34453.

27. Davies WH, Satter E, Berlin KS, Sato AF, Silverman AH, Fischer EA, et al. Reconceptualizing feeding and feeding disorders in interpersonal context: the case for a relational disorder. J Fam Psychol 2006;20:409-17.

28. Cardona Cano S, Tiemeier H, Van Hoeken D, Tharner A, Jaddoe VW, Hofman A, et al. Trajectories of picky eating during childhood: a general population study. Int J Eat Disord 2015;48:570-9.

29. Yi SH, Joung YS, Choe YH, Kim EH, Kwon JY. Sensory processing difficulties in toddlers with nonorganic failure-to-thrive and feeding problems. J Pediatr Gastroenterol Nutr 2015;60:819-24.

30. Broniatowski M, Sonies BC, Rubin JS, Bradshaw CR, Spiegel JR, Bastian RW, et al. Current evaluation and treatment of patients with swallowing disorders. Otolaryngol Head Neck Surg 1999;120:464-73.

31. Breton A, Eyoum I, Fournie-Gardini E, Olives JP, Vergeau D, Vignes M. Treatment of children with feeding disorders. Rev Laryngol Otol Rhinol (Bord) 2008;129:121-6. 


\section{Appendix 1.}

Feeding behavior disorders are related to food regulation difficulties based on physiological feelings of hunger or fullness [13]. The diagnostic criteria include organic and nonorganic causal factors, delays in oral motor skills and speech development, separation, and social reciprocity. Behavioral symptoms and diagnoses may differ based on the infant's developmental stage and include food refusal, food selectivity, poor reciprocity, and gagging. Chatoor [11] classified six subcategories.

\section{Diagnostic criteria}

\section{Feeding disorder of state regulation}

- The infant's feeding difficulties start in the first few months of life and are present for at least 2 weeks.

- The infant has difficulty reaching and maintaining a calm state of alertness for feeding.

- The infant fails to gain age-appropriate weight or may show less weight gain.

- The infant's feeding difficulties cannot be explained by a physical illness.

2. Feeding disorder of caregiver-infant reciprocity

- The feeding disorder is usually observed in the first year of life, when the infant presents with an acute medical problem (commonly an infection) to the primary care physician or the emergency room and the physician notices that the infant is malnourished.

- The infant shows lack of developmentally appropriate signs of social reciprocity (e.g., visual engagement, smiling, babbling) with the primary caregiver during feeding.

- The infant shows significant growth deficiency.

- The primary caregiver is often unaware of or in denial about the infant's feeding and growth problems.

- The growth deficiency and lack of relatedness are not due solely to a physical or pervasive developmental disorder.

3. Infantile anorexia

- Children refuse to eat adequate amounts of food for at least 1 month.

. Onset of food refusal often occurs during the transition to spoon and self-feeding, typically between 6 months and 3 years of age.

- The infant or toddler rarely communicates hunger, lacks interest in food and eating, and would rather play, walk, or talk than eat.

- The infant or toddler shows significant growth deficiency.

- Food refusal does not follow a traumatic event to the oropharynx or gastrointestinal tract.

. Food refusal is not due to an underlying medical illness.

\section{Sensory food aversion}

- This feeding disorder is characterized by the infant's or child's consistent refusal to eat certain foods with specific tastes, texture, temperatures, or smells for at least 1 month.

- The onset of the food refusal occurs during the introduction of a new or different types of food that is aversive to the child.

- The child's reaction to aversive food ranges from grimacing or spitting out the food to gagging and vomiting. Then, the child refuses to continue eating the food and frequently generalizes and refuses other foods with similar color, appearance, or smell. Consequently, the child may refuse whole food groups.

- The child is reluctant to try unfamiliar new foods but eats without difficulty when offered preferred foods.

- The child demonstrates specific dietary deficiencies without supplementation but usually does not show any growth deficiency and may even be overweight and/or 
- Displays oral motor and expressive speech delay and/or

- The preschool child becomes anxious during mealtime and avoids social situations that involve eating.

- Food refusal does not follow a trauma to the oropharynx.

- Refusal to eat specific foods is not related to a food allergy or any other medical illness.

\section{Posttraumatic feeding disorder}

- This feeding disorder is characterized by acute onset of severe and consistent food refusal.

- The onset of the food refusal can occur at any age from infancy to adulthood.

- Food refusal follows a traumatic event or repeated traumatic insults to the oropharynx or gastrointestinal tract that trigger intense distress in the child.

. Consistent refusal to eat manifests in one of the following ways, depending on the feeding (bottle or solid food) mode by the child in association with the traumatic event:

- Refuses to drink from a bottle but may accept food offered by spoon.

- Refuses solid foods but may accept a bottle, fluids, or pureed food.

- Refuses all oral feeding.

- Reminders of the traumatic event cause distress manifested by 1 or more of the following:

- Child shows anticipatory distress when positioned for feeding.

- Child shows intense resistance when approached with a bottle or food.

- Child shows resistance to swallowing food placed in the mouth.

- Food refusal poses acute and/or long-term threats to children's health, nutrition, and growth and threatens the progression of their age-appropriate feeding development.

\section{Feeding disorder associated with a concurrent medical condition}

- This feeding disorder is characterized by food refusal and inadequate food intake for at least 2 weeks.

- The onset of food refusal can occur at any age and may wax and wane in intensity depending on the underlying medical condition.

- The infant or toddler readily initiates feeding but shows distress and refuses to continue feeding over the course of a meal.

- The infant or toddler has a concurrent medical condition that is believed to cause distress.

- The infant or toddler fails to gain adequate weight or may lose weight.

- Medical management improves but may not fully alleviate the feeding problems. 